\title{
ADUBAÇÃO FOSFATADA E POTÁSSICA NO CRESCIMENTO E NUTRIÇÃO DA Crotalaria juncea L. ${ }^{1}$
}

\author{
Phosphate and potash fertilization on growth and nutrition of Crotalaria juncea L.
}

\author{
Kátia Aparecida de Pinho Costa 2 , Juarez Patrício de Oliveira Júnior ${ }^{3}$ Itamar Pereira de Oliveira ${ }^{4}$, \\ Alexandre Bryan Heinemann ${ }^{4}$, Valdemar Faquin ${ }^{5}$, Cristiane Rodrigues ${ }^{6}$
}

\begin{abstract}
RESUMO
O experimento foi conduzido em casa-de-vegetação, com objetivo de avaliar o efeito de doses de $\mathrm{P}$ e K na altura, produção de massa seca e nutrição da crotalária em ARGISSOLO VERMELHO-AMARELO-Eutrófico, sob vegetação do cerrado. O delineamento utilizado foi blocos ao acaso em esquema fatorial 4 x 4, com 3 repetições. Os tratamentos constituíram de quatro doses de $\mathrm{P}(0,25$, 50 e $75 \mathrm{~kg} \mathrm{ha}^{-1}$ de $\left.\mathrm{P}_{2} \mathrm{O}_{5}\right)$ e quatro de $\mathrm{K}\left(0,25,50\right.$ e $75 \mathrm{~kg} \mathrm{ha}^{-1}$ de $\left.\mathrm{K}_{2} \mathrm{O}\right)$. Aos 60 dias após a germinação, antes do período de floração, foi realizado o corte da leguminosa. Foram realizadas análises químicas da parte área para determinação das concentrações de macro e micronutrientes. A aplicação de $75 \mathrm{~kg} \mathrm{ha}^{-1}$ de $\mathrm{P}_{2} \mathrm{O}_{5}$ e $\mathrm{K}_{2} \mathrm{O}$, promoveu melhores resultados na altura de planta e produção de massa seca da crotalária. As maiores concentrações de $\mathrm{P}$ e Ca foram observadas com as doses de $75 \mathrm{~kg} \mathrm{ha}^{-1} \mathrm{de}_{2} \mathrm{O}_{5}$ e a maior concentração de $\mathrm{K}$ foi obtida com a aplicação de $75 \mathrm{~kg} \mathrm{ha}^{-1}$ de $\mathrm{K}_{2} \mathrm{O}$.
\end{abstract}

Termos para indexação: Nutrição mineral, produção de massa seca.

\begin{abstract}
The experiment was carried under greenhouse conditions with the objective of evaluating P and K effects on plant height, dry matter production and nutrition of crotalaria in an eutrophic Red - Yellow Argisol (alfisol), under cerrado vegetation. The experimental design was a randomized blocks in a $4 \mathrm{x} 4$ factorial scheme, with three replications. The treatments involved four phosphorus levels $\left(0,25,50\right.$, and $75 \mathrm{~kg} / \mathrm{ha}$ of $\left.\mathrm{P}_{2} \mathrm{O}_{5}\right)$ and four potassium levels $\left(0,25,50\right.$, and $75 \mathrm{~kg} / \mathrm{ha}$ of $\left.\mathrm{K}_{2} \mathrm{O}\right)$. At sixty days, after sowing and before flowering period, plants were harvested. Shoot's macro and micronutrient concentrations were determined by chemical analysis. Application of $75 \mathrm{~kg} \mathrm{ha}^{-1} \mathrm{P}_{2} \mathrm{O}_{5}$ and $\mathrm{K}_{2} \mathrm{O}$ promoted the best reults in plant height and dry matter production of crotalaria. Highest $\mathrm{P}$ and $\mathrm{Ca}$ concentrations were observed by applying $75 \mathrm{~kg} \mathrm{ha}^{-1}$ of $\mathrm{P}_{2} \mathrm{O}_{5}$ and the highest $\mathrm{K}$ concentration was verified when $75 \mathrm{~kg} \mathrm{ha}^{-1}$ of $\mathrm{K}_{2} \mathrm{O}$ were applied.
\end{abstract}

Index terms: Mineral nutrition, dry mass production.

(Recebido para publicação em 8 de setembro de 2005 e aprovado em 18 de abril de 2006)

\section{INTRODUÇÃO}

O uso de adubos nitrogenados nem sempre é economicamente viável, devido ao seu custo elevado e baixo índice de aproveitamento. Desta forma, as leguminosas constituem melhor aproveitamento do processo de fixação biológica do $\mathrm{N}$ atmosférico $\left(\mathrm{N}_{2}\right)$, sendo considerada uma das opções mais viáveis para se reduzir o custo de produção e o risco de poluição ambiental provocada pelos adubos nitrogenados (SOUZA \& LOBATO, 2004).

Apesar da importância da adubação verde, essa prática conservacionista é ainda pouco utilizada e estudada nos solos sob cerrado, necessitando-se, assim de informações básicas como: espécies mais adequadas, característica fenológicas e exigência dessas espécies.
Atualmente, dentre as diversas leguminosas usadas para adubação verde na região do cerrado destaca-se as espécies mais promissoras (AMABILE, 1996). A crotalária é uma leguminosa muito usada em rotação com diversas culturas e no enriquecimento do solo, por apresentar grande potencial de fixação biológica de $\mathrm{N}$. Apresenta fácil e abundante nodulação com espécies de rizóbio de crescimento lento. $\mathrm{O}$ aporte de $\mathrm{N}$ ao sistema solo-planta é estimado entre 100 e $300 \mathrm{~kg} \mathrm{ha}^{-1} \mathrm{ano}^{-1}$ de N (LOVERA, 2003).

Myasaka (1984) relata que a crotalária é uma leguminosa anual de porte alto, podendo alcançar mais de dois metros de altura. Apresenta rápido crescimento, sendo muito competitiva com plantas daninhas. Segundo Silva (2002), essa leguminosa proporciona uma boa cobertura do solo, chegando a produzir $15.201 \mathrm{~kg} \mathrm{ha}^{-1}$ de massa seca.

\footnotetext{
'Trabalho Realizado na Escola de Agronomia da UFG.

${ }^{2}$ Doutoranda em Solos e Nutrição de Plantas, Bolsista CNPQ, Departamento de Ciência do Solo, Universidade Federal de Lavras/UFLA - Cx. P. 3037 37200-000 - Lavras, MG - katiazoo@hotmail.com .

3Professor Dr., Escola de Agronomia da Universidade Federal de Goiás/UFG

${ }^{4}$ Pesquisadores da Embrapa Arroz e Feijão - itamar@cnpaf.embrapa.br

${ }^{5}$ Professor titular - Departamento de Ciência do Solo - Universidade Federal de Lavras/UFLA - Cx. P. 3037 - 37200-000 - Lavras, MG - vafaquin@ufla.br

${ }^{6}$ Aluna de Pós-Graduação da Universidade Federal de Goiás/UFG.
} 
Em condições de cerrado, a crotalária constitui uma excelente leguminosa para o sistema agrícola, levando-se em consideração o retorno indireto, ou seja, os benefícios gerados às culturas complementares, como reciclagem de nutrientes, aumento da matéria orgânica (AMABILE et al., 2000). Essa leguminosa pode ser ainda empregada como forragem na alimentação de bovinos/suínos, sendo que o primeiro corte poderá ser feito aos 45-60 dias e, posteriormente, de $30 \mathrm{em} 30$ dias, não sendo recomendado o corte quando surgirem os botões florais (CALEGARI, 1995).

A Crotalaria juncea comporta-se bem em solos argilosos e arenosos com razoáveis níveis de fertilidade, por isso é de suma importância manter os solos em níveis ideais de fertilidade para a planta (CALEGARI, 1995). A disponibilidade de $\mathrm{P}$ na região do cerrado, em condições naturais é muito baixa. Dessa forma a adubação fosfatada é uma prática imprescindível, por ser um nutriente muito importante nos primeiros dias de vida da planta, sendo determinante para o sucesso no estabelecimento e nenhum outro nutriente pode substituí-lo. A planta precisa do fósforo para completar seu ciclo normal de produção (LOPES, 1989).

Outro nutriente importante para as leguminosas é o K, que segundo Mengel \& Kirkby (1987), é um elemento essencial para todos os organismos vivos, sendo o cátion mais importante nos processos fisiológicos das plantas, não somente pela sua concentração nos tecidos vegetais, mas também pelas suas funções bioquímicas. A exigência de K para o ótimo crescimento das plantas varia entre 20 a $50 \mathrm{~g} \mathrm{~kg}^{-1}$ de massa seca do vegetal. Este nutriente está presente nas plantas na forma de $\mathrm{K}^{+}$e é altamente móvel. Tem ação fundamental no metabolismo vegetal, pelo papel que exerce na fotossíntese, atuando no processo de transformação da energia luminosa em energia química, sendo um nutriente mineral retirado em grande quantidade pelas plantas (MARSCHNER, 1995).

Segundo Menezes (2002), as plantas forrageiras deficientes em $\mathrm{K}$ apresentam colmos finos, raquíticos e pouco resistentes ao acamamento, as folhas são pouco desenvolvidas e em fase mais avançada de deficiência ocorre a clorose e posterior necrose nas pontas e nas margens, afetando assim a produtividade da planta forrageira. Raij (1991) também enfatizou que as plantas em condições de carência deste nutriente apresentam turgidez reduzida, e sob deficiência de água tornam-se flácidas, sendo pouco resistentes à seca e mais susceptíveis ao ataque de fungos.

Conduziu-se este trabalho com o objetivo de avaliar o efeito do $\mathrm{P}$ e $\mathrm{K}$ na altura, produção de massa seca e nutrição mineral da Crotalaria juncea, através da concentração foliar de N, P, K, Ca, Mg, K, Cu, Fe Mn e Zn.

\section{MATERIAL E MÉTODOS}

O experimento foi realizado em casa-de-vegetação da Escola de Agronomia da Universidade Federal de Goiás. Foram coletas amostras de solo na profundidade de 0-20 $\mathrm{cm}$, classificado como ARGISSOLO VERMELHOAMARELO-Eutrófico, com as seguintes características: pH em água: 6,1, Ca: $3,4 \mathrm{cmol}_{\mathrm{c}} \mathrm{dm}^{-3} ; \mathrm{Mg}: 1,5 \mathrm{cmol}_{\mathrm{c}} \mathrm{dm}^{-3} ; \mathrm{Al}$ : $0,0 \mathrm{cmol}_{\mathrm{c}} \mathrm{dm}^{-3} ; \mathrm{Al}+\mathrm{H}: 1,7 \mathrm{cmol}_{\mathrm{c}} \mathrm{dm}^{-3} ; \mathrm{P}: 3,7 \mathrm{mg} \mathrm{dm}^{-3} ; \mathrm{K}: 27$ $\mathrm{mg} \mathrm{dm}{ }^{-3} ; \mathrm{S}: 3,1 \mathrm{mg} \mathrm{dm}^{-3} ; \mathrm{Cu}: 3,1 \mathrm{mg} \mathrm{dm}^{-3} ; \mathrm{Zn}: 1,1 \mathrm{mg} \mathrm{dm}^{-3}$; Fe: $46,7 \mathrm{mg} \mathrm{dm}^{-3}$; Mn: 57,8 $\mathrm{mg} \mathrm{dm}^{-3}$; CTC: 6,66 $\mathrm{cmol}_{\mathrm{c}} \mathrm{dm}^{-3}$, V: $74,47 \%$; M.O: $2,3 \%$. A metodologia utilizada para a análise de solo foi da Embrapa (1997).

Foram utilizados vasos com $3 \mathrm{~kg}$ de terra. $\mathrm{O}$ delineamento utilizado foi blocos ao acaso em esquema fatorial $4 \times 4$, com 3 repetições. Os tratamentos constituíram de quatro doses de $\mathrm{P}\left(0,25,50\right.$ e $75 \mathrm{~kg} \mathrm{ha}^{-1}$ de $\mathrm{P}_{2} \mathrm{O}_{5}$, equivalentes a $\left.0 ; 5,5 ; 11,0 ; 16,5 \mathrm{mg} \mathrm{dm}^{-3}\right)$ e quatro de $\mathrm{K}(0$, 25,50 e $75 \mathrm{~kg} \mathrm{ha}^{1}$ de $\mathrm{K}_{2} \mathrm{O}$, equivalentes a $0 ; 10 ; 20$ e $40 \mathrm{mg}$ $\left.\mathrm{dm}^{-3}\right)$. As fontes utilizadas foram o superfosfato simples e cloreto de potássio.

As sementes foram aplicadas junto com o $\mathrm{P}$ na profundidade de $2 \mathrm{~cm}$. Dez dias após a germinação, o K foi aplicado em cobertura. Foi realizado um desbaste que permitiu a permanência de três plantas por vaso.

Aos 60 dias após a germinação, antes do período de floração, foi medida a altura das plantas com o auxílio de uma régua, e em seguida foi realizado o corte da leguminosa a $5 \mathrm{~cm}$ da superfície do solo. $\mathrm{O}$ material coletado foi acondicionado em saco de papel, identificado, pesado e colocado em estufa de ventilação forçada de ar, com temperaturas de 58 a $65^{\circ} \mathrm{C}$ por 72 horas, para determinação da matéria seca parcial (SILVA \& QUEIROZ, 2002).

A análise química foi realizada no Laboratório de Nutrição de Plantas da Embrapa Arroz e Feijão, para determinação dos minerais: Macronutrientes: nitrogênio $(\mathrm{N})$, fósforo $(\mathrm{P})$, enxofre $(\mathrm{S})$, potássio $(\mathrm{K})$, cálcio $(\mathrm{Ca})$, magnésio $(\mathrm{Mg})$ e Micronutrientes: zinco $(\mathrm{Zn})$, cobre $(\mathrm{Cu})$, ferro $(\mathrm{Fe})$ e manganês $(\mathrm{Mn})$. $\mathrm{O} \mathrm{N}$ foi determinado pelo método de Kjeldahl, o $\mathrm{S}$ foi determinado por turbidimetria do sulfato de bário, o P por colorimetria do metavanadato, o K, por fotometria de chama de emissão e as concentrações de $\mathrm{Ca}, \mathrm{Mg}, \mathrm{Zn}, \mathrm{Cu}, \mathrm{Mn}$ e $\mathrm{Fe}$ por espectrofotometria de absorção atômica, como relatados por Malavolta et al. (1997).

Os resultados foram analisados através do procedimento GLM do programa estatístico SAS Institute (1989), em que as médias foram submetidas à análise de regressão. 


\section{RESULTADOS E DISCUSSÃO}

Houve efeito significativo $(P<0,01)$ na interação de doses de $\mathrm{P}$ x K. A crotalária respondeu à adubação fosfatada e potássica na altura de planta e produção de massa seca (MS). De acordo com a superfície de resposta o valor máximo de altura de planta e produção de MS ocorreu nas doses de $75 \mathrm{~kg} \mathrm{ka}^{-1}$ de $\mathrm{P} \mathrm{e} \mathrm{K}$, equivalente a aumentos em relação à testemunha de 53,3 e 46,5\%, respectivamente. Estas constatações salientam a importância da adubação fosfatada e potássica para boa produção de MS de forragem, mostrando ser uma leguminosa exigente por esses nutrientes. Wrolow et al. (2004a), avaliando o efeito de fósforo e potássio sobre o desenvolvimento de três leguminosas, observaram efeito significativo apenas para doses de $\mathrm{P}$ aplicadas, sendo que o potássio não influenciou na produção de MS das leguminosas. Oliveira et al. (2001) relatam que o P é considerado um dos nutrientes mais importantes no desenvolvimento das plantas, melhorando a produção de massa seca e, conseqüentemente, a qualidade da forragem.

Observa-se na Figura 2 que em condições de baixas doses de $\mathrm{K}$ mesmo com o incremento das doses de $\mathrm{P}$ a produção de MS permanecem constante, mas no entanto, à medida que se aumentou a dose de $\mathrm{K}$ associada às doses de $\mathrm{P}$ a produção foi incrementada.

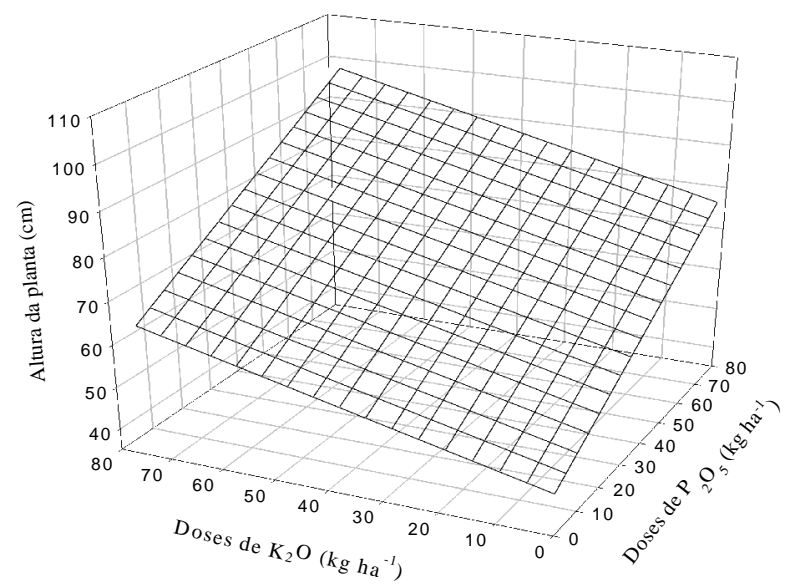

FIGURA 1 - Efeito de doses de P e K na altura de planta da crotalária.

Avaliando as concentrações de nutrientes na parte aérea da planta, observa-se nas Figuras 3 e 4 que houve efeito significativo $(\mathrm{P}<0,05)$ de doses $\mathrm{P}$ para as concentrações de $\mathrm{P}$ e Ca, mostrando um aumento de 52,66 e $58,75 \%$, respectivamente, em relação à testemunha. Contudo, não foram observados efeitos significativos $(\mathrm{P}>0,05)$ de doses de $\mathrm{K}$ e na interação P x K. A absorção de P e Ca na parte área da crotalária foi proporcional à quantidade de fertilizante fosfatado aplicado. As concentrações desses nutrientes aumentaram de forma linear até $75 \mathrm{~kg} \mathrm{ha}^{-1}$ de $\mathrm{P}_{2} \mathrm{O}_{5}$ chegando atingir concentrações de $10,8 \mathrm{~g} \mathrm{~kg}^{-1}$ de $\mathrm{P}$ e $14,9 \mathrm{~g}$ $\mathrm{kg}^{-1}$ de $\mathrm{Ca}$. Pode-se atribuir esse efeito à capacidade das leguminosas fixar e absorver grandes quantidades de $\mathrm{N}$, necessitando, com isso concentrações proporcionais de $\mathrm{P}$ para balanço de absorção desses nutrientes pela planta. Boa parte do $\mathrm{Ca}$ absorvido encontra-se na parede celular na forma de sal orgânico dos ácidos orgânicos da lamela média (MALAVOLTA, 1976).

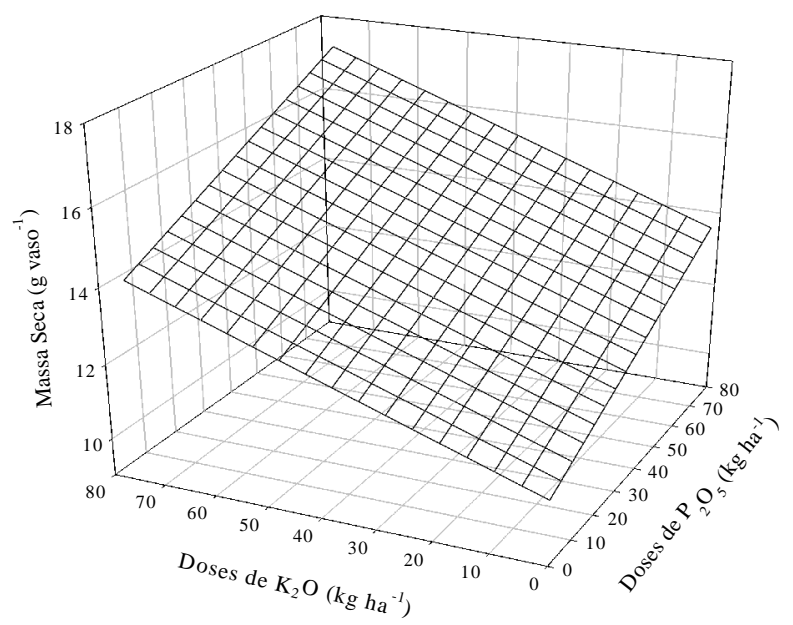

FIGURA 2 - Efeito de doses de fósforo e potássio na produção de massa seca da crotalária.

Machado et al. (2004), trabalhando com quatro doses de $\mathrm{P}$ e de $\mathrm{K}\left(0,30,60\right.$ e $90 \mathrm{~kg} \mathrm{ha}^{-1}$ de $\mathrm{P}_{2} \mathrm{O}_{5}$ e $\mathrm{K}_{2} \mathrm{O}$, respectivamente) no amendoim forrageiro (Arachis pintoi Krap. et Greg. cv. Amarillo), verificaram efeito significativo na concentração de $\mathrm{P}$, mostrando uma resposta linear às doses deste nutriente apresentando teor máxima na matéria seca de $15,96 \mathrm{~g} \mathrm{~kg}^{-1}$.

Houve efeito significativo $(P<0,01)$ das doses de $K$ nas concentrações desse nutriente na parte área. A concentração máxima obtida de $\mathrm{K}\left(12,8 \mathrm{~g} \mathrm{~kg}^{-1}\right)$ foi verificada com a aplicação de $75 \mathrm{~kg} \mathrm{ha}^{-1}$ de $\mathrm{K}_{2} \mathrm{O}$, apresentando uma resposta linear em relação à quantidade de fertilizante potássico aplicado (Figura 5). Contudo não foram verificados efeitos significativos $(\mathrm{P}>0,05)$ de doses de $\mathrm{P}$ e na interação P x K. Resultados semelhantes foram obtidos 
por Wrolow et al. (2004b) que observaram efeito significativo na concentração de $\mathrm{K}$ nas doses de $\mathrm{K}$ aplicadas, atingindo valores entre 8,0 e $16,0 \mathrm{~g} \mathrm{~kg}^{-1}$.

Concentrações maiores de nutrientes na crotalária foram observadas por Meneses (2002), que ao avaliar a fitomassa de diferentes coberturas, com potencial de utilização em sistema de plantio direto, encontrou valores de $\mathrm{P}, \mathrm{K}$ e Ca de 17,0; 17,0; 16,0 $\mathrm{g} \mathrm{kg}^{-1}$, respectivamente. Esses resultados podem ser atribuídos ao plantio direto, que devido à cobertura superficial com restos culturais, proporcionou maiores teores de água. Com isso, foi obtidas melhores solubilidades dos sais e carreamento dos nutrientes no solo, fazendo com que maiores concentrações de nutrientes ficassem em contato com o sistema radicular da leguminosa, facilitando a absorção

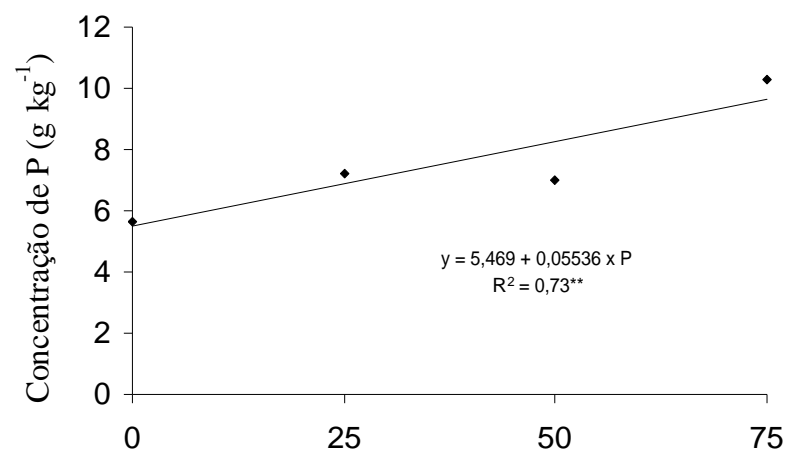

Doses de $\mathrm{P}_{2} \mathrm{O}_{5}\left(\mathrm{~kg} \mathrm{ha}^{-1}\right)$

FIGURA 3 - Concentração de $\mathrm{P}$ na parte área da crotalária em função de doses de $\mathrm{P}_{2} \mathrm{O}_{5}$.

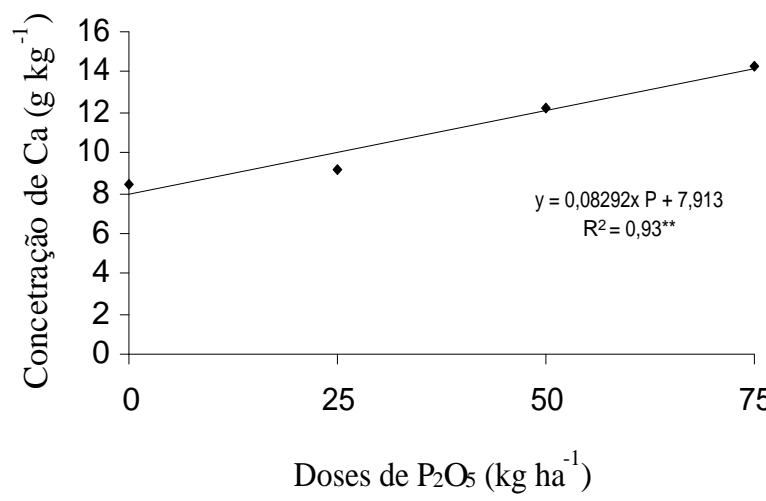

FIGURA 4 - Concentração de Ca na parte área da crotalária em função de doses de $\mathrm{P}_{2} \mathrm{O}_{5}$. dos nutrientes pelas plantas. Possivelmente, o cultivo tradicional da crotalária realizado nesta pesquisa, não tenha proporcionado condições favoráveis para facilitar o processo de absorção.

Não foram observados efeitos significativos (P> $0,05)$ de doses de $\mathrm{P}$ e $\mathrm{K}$, e na interação $\mathrm{P} \times \mathrm{K}$, nas concentrações de N, S, Mg, Cu, Zn, Mn e Fe. De acordo com esses resultados, observa-se que as quantidades de P e K aplicadas não elevaram ao máximo a produção da crotalária. Caso doses maiores de fertilizantes fossem usadas, a curva de produção atingiria um máximo, e em seguida uma queda no rendimento da leguminosa e com isso, possivelmente interações poderiam ter sido observadas, caso ocorressem efeitos quadráticos da doses de fertilizantes na absorção de nutrientes.

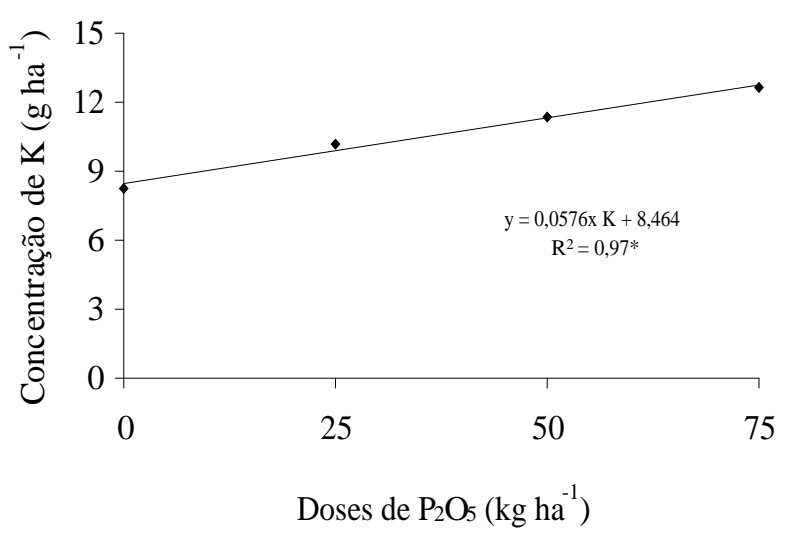

FIGURA 5 - Concentração de K na parte área da crotalária em função de doses de $\mathrm{K}_{2} \mathrm{O}$.

\section{CONCLUSÃO}

A aplicação de $75 \mathrm{~kg} \mathrm{ha}^{-1}$ de $\mathrm{P}_{2} \mathrm{O}_{5}$ e $\mathrm{K}_{2} \mathrm{O}$, promoveu melhores resultados na altura de planta e produção de massa seca da crotalária, equivalente a aumentos em relação à testemunha de 53,3 e 46,5\%, respectivamente.

As maiores concentrações de $\mathrm{P}$ e $\mathrm{Ca}$ foram observadas com as doses de $75 \mathrm{~kg} \mathrm{ha}^{-1}$ de $\mathrm{P}_{2} \mathrm{O}_{5}$ e a maior concentração de $\mathrm{K}$ foi obtida com a aplicação de $75 \mathrm{~kg} \mathrm{ha}^{-1}$ de $\mathrm{K}_{2} \mathrm{O}$. Em relação ao $\mathrm{P}$, essa dose resultou em aumento de 52,66 e 58,75\% na concentração de P e Ca na folha, respectivamente. A dose de $\mathrm{K}$ para atingir o máximo de produção resultou em aumento de $65,06 \%$ na absorção desse nutriente.

Ciênc. agrotec., Lavras, v. 30, n. 5, p. 827-831, set./out., 2006 


\section{REFERÊNCIAS BIBLIOGRÁFICAS}

AMABILE, R. F. Comportamento de adubos verdes em épocas de semeadura nos cerrados do Brasil Central. 1996. 123 f. Dissertação (Mestrado em Agronomia) - Escola Superior de Agricultura "Luiz de Queiroz", Universidade de São Paulo, Piracicaba, 1996.

AMABILE, R. F.; FANCELLI, A. L.; CARVALHO, A. M. Comportamento de espécies de adubos verdes em diferentes épocas de semeadura e espaçamentos na região dos Cerrados. Pesquisa Agropecuária Brasileira, Brasília, v. 35, p. 47-54, 2000.

CALEGARI, A. Leguminosas para adubação verde de verão no Paraná. Londrina: IAPAR, 1995. 118 p. (Circular, 80).

EMPRESA BRASILEIRA DE PESQUISA AGROPECUÁRIA. Centro Nacional de Pesquisa de Solos. Ministério da Agricultura e do Abastecimento. Manual de métodos de análise de solo. 2. ed. rev. e atual. Rio de Janeiro, 1997. 212 p.

LOPES, A. S. Manual de fertilidade do solo. Piracicaba: Associação Brasileira para Pesquisa da Potassa e do Fosfato, 1989. 155 p.

LOVERA, B. T. F. Estabelecimento do amendoim forrageiro (Arachis pintoi) cv. Amarillo em associação com milho (Zea mays). 2003. 56 f. Dissertação (Mestrado em Zootecnia - Pastagem) - Universidade Federal de Pelotas, Pelotas, 2003.

MACHADO, A. N.; FERREIRA, O. G. L.; SIEWERDT, L.; AFFONSO, A. B. Fósforo e potássio na qualidade da forragem de Arachis pintoi introduzida em campo natural, RS. In: REUNIÃO ANUAL DA SOCIEDADE BRASILEIRA DE ZOOTECNIA, 41., 2004, Campo Grande. Anais... Campo Grande: SBZ, 2004. CD-ROM.

MALAVOLTA, E. Manual de química agrícola: nutrição de plantas e fertilidade do solo. São Paulo: Agronômica Ceres, 1976.

MALAVOLTA, E.; VITTI, G. C.; OLIVEIRA, S. A. Avaliação do estado nutricional das plantas: princípios e aplicações. 2. ed. Piracicaba: Associação Brasileira da Potassa e do Fosfato, 1997. 319 p.

MARSCHNER, H. Mineral nutrition of higher plants. 2. ed. London: Academic, 1995. 889 p.
MENEZES, L. A. S. Alteração de propriedades químicas e físicas do solo em função da fitomassa de plantas de cobertura. 2002. 73 f. Dissertação (Mestrado em Agronomia) - Universidade Federal de Goiás, Goiânia, 2002.

MENGEL, K.; KIRKBY, E. Principles of plant nutrition. 4. ed. Bern: International Potash Institute, 1987. 687 p.

MYASAKA, S. Histórico de estudos de adubação verde, leguminosas viáveis e suas características. In: FUNDAÇÃO CARGILL. Adubação verde no Brasil. Campinas, 1984. p. 30-33.

OLIVEIRA, I. P.; CASTRO, F. G. F.; MOREIRA, F. P.; PAIXÃO, V. V.; CUSTÓDIO, D. P.; SANTOS, R. S. M.; FARIA, C. D. Efeitos qualitativo e quantitativo da aplicação do zinco no capim Tanzânia-1. Pesquisa Agropecuária Tropical, Goiânia, v. 20, n. 1, p. 43-48, 2001.

RAIJ, V. Fertilidade do solo e adubação. São Paulo: Ceres; Associação Brasileira para Pesquisa da Potassa e do Fosfato, 1991. 343 p.

SAS INSTITUTE. Language and procedures. Version 6. Cary, 1989.

SILVA, D. J.; QUEIROZ, A. C. Análise de alimentos: métodos químicos e biológicos. 3. ed. Viçosa: UFV, 2002. 235 p.

SILVA, T. R. B. Adubação nitrogenada e resíduos vegetais no desenvolvimento do feijoeiro (Phaseolus vulgares $L$.) em sistema de plantio direto. 2002. 79 f. Dissertação (Mestrado em Agronomia) - Universidade Estadual Paulista, Ilha Solteira, 2002.

SOUZA, D. M. G.; LOBATO, E. Cerrado: correção do solo e adubação. 2. ed. Brasília, DF: Embrapa Informações Tecnológicas, 2004. 416 p.

WROLOW, R. H.; MISTURE, C.; COELHO, R. W.; SIEWERDT, L.; ZONTA, E. P. Efeito do fósforo e do potássio sobre o desenvolvimento e a nodulação de três leguminosas anuais de estação fria. Revista Brasileira de Zootecnia, Viçosa, v. 33, n. 6, p. 2224-2230, 2004a.

WROLOW, R. H.; MISTURE, C.; COELHO, R. W.; SIEWERDT, L.; ZONTA, E. P. Composição bromatológica de três leguminosas anuais de estação fria adubadas com fósforo e potássio. Revista Brasileira de Zootecnia, Viçosa, v. 33, n. 6, p. 2231-2239, 2004b. 